\title{
A Novel Speckle Tracking Technique for Investigating Regional Motion of the Carotid Wall: Spatio-temporal Variation in Distension Associates with Presence of Calcified Plaque
}

\author{
Wang $\mathrm{J}^{1}$, Spector $\mathrm{T}^{2}$, Chowienczyk $\mathbf{P J}^{3,4 *}$ and Jiang $\mathrm{B}^{3}$ \\ ${ }^{1}$ Department of Ultrasound, Union Hospital, Tongji Medical College, Huazhong University of Science and Technology, Wuhan, China \\ ${ }^{2}$ Department of Twin Research, King's College, London, UK \\ ${ }^{3}$ King's College, London British Heart Foundation Centre, Department of Clinical Pharmacology, London, UK \\ ${ }^{4}$ Department of Clinical Pharmacology, St Thomas' Hospital, Lambeth Palace Road, London SE1 7EH, UK
}

\begin{abstract}
Arterial calcification may lead to regional variation in distension imposing stresses on the arterial wall that predispose to plaque rupture. The objective of this study was to use a novel speckle tracking method to investigate regional motion of the carotid wall and to determine whether this relates to subclinical disease. Measurements were obtained on 256 subjects from the Twins UK cohort (mean \pm SD age $62 \pm 10.2$ years). The left carotid was imaged for an assessment of plaque and calcification. Speckle-tracking was then used to measure regional circumferential strain of the left common carotid in 6 separate $60^{\circ}$ segments of the circumference of the arterial wall in a plaque free plane of the common carotid approximately $1 \mathrm{~cm}$ proximal to the bifurcation. Regional variation in circumferential strain around the circumference of the arterial wall was characterized by the standard deviation of circumferential strain and that of the time from onset of systole to peak circumferential strain in each segment. Spatio-temporal variation in circumferential strain characterized by variation in the time to peak circumferential strain was associated with age and presence of calcified plaque (regression coefficients 0.73 units/year and 14.2 increase for presence of calcified plaque, each $\mathrm{P}<0.001$ ) independent of other confounding factors and of other measures of arterial wall damage such as carotid intima-media thickening, carotid distensibility and carotid-femoral pulse wave velocity. Arterial ageing and calcification are associated with spatio-temporal variation in distension of the carotid artery.
\end{abstract}

Keywords: Carotid artery; Ultrasound; Speckle-tracking; Circumferential strain; Spatio-temporal variation; Atherosclerotic plaque

\section{Introduction}

Ultrasound imaging of the carotid artery is widely used in the evaluation of arterial health. Intima-media thickening and presence of atherosclerotic plaque are both associated with increased risk of coronary events and stroke but the presence of plaque, particularly calcified plaque, confers greater risk [1-4]. Arterial stiffening has also received much attention because it may precede structurally apparent change in the arterial wall. However, although carotid-femoral pulse wave velocity (PWV), an integrated measure of stiffness mainly along the aorta is a measure of risk of CVD events [5], measures of carotid artery distensibility do not appear predict risk in the general population [6,7]. Pathological change in the arterial wall particularly that associated with atherosclerotic plaque is highly variable in its distribution. This might be expected to lead to regional variation in distension of the arterial wall. Such variation could impose high levels of wall stress predisposing to plaque rupture [8-10] and could be a useful marker of risk of cardiovascular events associated with subclinical disease. Here we describe application of 2-D speckle tracking, developed to study regional wall motion in the heart to characterise spatio-temporal variation in distension of the common carotid artery. We used this to examine the relation of variation in wall movement to risk factors and presence of calcified plaque, calcification being a focal process expected to lead to regional variation in stiffening and known to be associated with a high risk of cardiovascular events [3].

\section{Methods}

\section{Study population}

Two hundreds and fifty-six asymptotic individuals (mean \pm SD age of $62 \pm 10.2$ years) were enrolled from a study on vascular ageing in the Twins UK cohort. This is a cohort of predominantly female middle-aged subjects with lifestyle characteristics such as smoking and cardiovascular risk factors similar to that of the general population. The study included measurement of biochemical risk factors, central and peripheral blood pressure, carotid-femoral pulse wave velocity (cfPWV) and measurement of carotid wall properties. The study was approved by St Thomas' Hospital Research Ethics Committee, and written informed consent was obtained from all subjects. The sub-sample of Twins UK participating in the present study was consecutively recruited subjects over the period from Dec.2012 to Feb.2014. A sample size of $>250$ was estimated (from previous statistics on Twins UK) to provide at least 50 subjects with calcified plaque and to allow a difference of $0.5 \mathrm{SD}$ in measures of strain to be detected between subject with and without calcified plaque.

\section{Blood pressure and pulse wave velocity}

Measurements were performed in a quiet temperature-controlled vascular laboratory $\left(22^{\circ} \mathrm{C}-24^{\circ} \mathrm{C}\right)$. Brachial blood pressure was

*Corresponding author: Phil Chowienczyk, Professor, King's College, London British Heart Foundation Centre, Department of Clinical Pharmacology, London, UK, SE1 7EH UK, Tel: 44207177 4642; Fax: 44207 4012242; E-mail: phil.chowienczyk@kcl.ac.uk

Received November 16, 2015; Accepted November 24, 2015; Published December 10, 2015

Citation: Wang J, Spector T, Chowienczyk PJ, Jiang B (2015) A Novel Speckle Tracking Technique for Investigating Regional Motion of the Carotid Wall: Spatiotemporal Variation in Distension Associates with Presence of Calcified Plaque. J Vasc Med Surg 3: 233. doi:10.4172/2329-6925.1000233

Copyright: (c) 2015 Wang J, et al. This is an open-access article distributed unde the terms of the Creative Commons Attribution License, which permits unrestricted use, distribution, and reproduction in any medium, provided the original author and source are credited. 
Citation: Wang J, Spector T, Chowienczyk PJ, Jiang B (2015) A Novel Speckle Tracking Technique for Investigating Regional Motion of the Carotid Wall: Spatio-temporal Variation in Distension Associates with Presence of Calcified Plaque. J Vasc Med Surg 3: 233. doi:10.4172/23296925.1000233

Page 2 of 4

measured in duplicate, with a validated oscillometric device (Omron 705CP, Omron, Tokyo, Japan) after subjects had rested supine for at least 10 min. Radial tonometry was performed using the SphygmoCor system (Atcor Medical, Sydney, Australia) and central blood pressure components obtained using the SphygmoCor transfer function with calibration from brachial systolic and diastolic blood pressures. cfPWV was measured using sequential carotid/femoral ECG referenced applanation tonometry with the SphygmoCor system. The path distance was estimated from the distance between the sternal notch and femoral artery at the point of applanation. At least 2 sequential measurements meeting the inbuilt quality control criteria of the SphygmoCor system were obtained and the mean value used for analysis.

\section{D ultrasonography}

The carotid arteries were scanned by GE VIVID7 scanner with 12 $\mathrm{MHz}$ linear transducer. Arterial walls were examined for plaque in the common carotids, at the carotid bifurcations and origins of the internal and external carotid arteries. Plaque was defined in the longitudinal view as focal widening and protrusion into the lumen of $\geq 1.5 \mathrm{~mm}$ thickness relative to neighboring areas and confirmed in transverse view. Plaque was graded according to echogenicity into predominately echolucent/noncalcified ( $>50 \%$ of similar echogenicity to blood), as a soft plaque, or echogenic/calcified ( $>50 \%$ of similar echogenicity to the bright echo of the media-adventitia interface, as a hard plaque).

Optimal dynamic long and short axis images were acquired in a plaque free segment of the common carotid artery $1-2 \mathrm{~cm}$ proximal to the carotid bulb, using a high frame rate (65 frames/second). Three cardiac cycles were stored in a cine loop for subsequent post-processing. Common carotid intima-media thickness (IMT) was determined using computer semi-automated edge detective software (Medical Imaging Applications, Coralville, Iowa). Mean values of IMT of the near and far walls were used for analysis.

\section{Two dimensional speckle tracking analysis of carotid wall distension}

Two dimensional speckle tracking analysis (2D-ST) was performed in a plaque free segment of the left common carotid at a point approximately $1 \mathrm{~cm}$ proximal to the carotid bulb. Short axis (cross-plane) images were acquired at a frame rate of 65 frames/second) over three cardiac cycles. Speckle-tracking technology developed to determine regional myocardial wall strain (EchoPac, version 7.0, GE Healthcare,
Norway) was used to measure regional circumferential strain of the common carotid wall. The intima of the artery was outlined manually at the end of systole and the speckle tracking software used to trace circumferential strain in 6 separate $60^{\circ}$ segments of the circumference of the arterial wall (Figure 1). Peak systolic circumferential strain $\left(\mathrm{CS}_{\mathrm{p}}\right)$ and time of peak circumferential strain $\left(\mathrm{CS}_{\mathrm{tp}}\right)$ where recorded for each of the $60^{\circ}$ segments. The standard deviations (SD) of these values over the 6 segments were used as measures of variation in peak circumferential distension (VCS $)$, and time of peak circumferential distension $\left(\mathrm{VCS}_{\mathrm{tp}}\right)$ respectively. A sub-sample of 20 subjects selected at random were scanned and measured on two occasions to assess the repeatability of the measures $\mathrm{VCS}_{\mathrm{p}}$ and $\mathrm{VCS}_{\mathrm{tp}}$.

\section{Statistical analysis}

Subject characteristics are presented as means \pm SD; all other results are presented as means \pm SE. Subject characteristics were compared with Student's unpaired $t$-test or by Chi-squared test for categorical values. The relation between measures of variation in circumferential strain $\left(\mathrm{VCS}_{\mathrm{p}}\right.$ and $\left.\mathrm{VCS}_{\mathrm{tp}}\right)$ with cardiovascular risk factors, carotid IMT and $\mathrm{cfPWV}^{\mathrm{p}}$ was explored by univariate and multivariate regression analysis (with dummy variables for categorical values). Blood pressure components included in the primary analysis were mean arterial pressure (MAP) and central pulse pressure since strain is proportional to pulse pressure (and distensibility). Multivariate regression analysis was done by forcing all confounding variables into the model and by backward stepwise elimination. Mean values of $\mathrm{CS}_{\mathrm{p}}$ and $\mathrm{CS}_{\mathrm{tp}}$ (for VCS and $\mathrm{VCS}_{\mathrm{tp}}$ respectively) were included in all regression models (but were allowed to step out of backward models) to account for variation being proportional to mean values. Analysis of covariance (ANCOVA) was used to compare $\mathrm{VCS}_{\mathrm{p}}$ and $\mathrm{VCS}_{\mathrm{tp}}$ in subjects with and without calcified and non-calcified carotid artery plaque with adjustment for age, sex and risk factors. IBM SPSS statistical software version 21 (SPSS Inc., UK) was used for all analysis. $\mathrm{P}<0.05$ was considered significant.

\section{Results}

Subject characteristics are summarized in Table 1. Typical circumferential strain curves for each of the 6 segments of the circumference of the common carotid are shown in Figure 1. In young subjects with plaque free walls, strain curves were similar in each segment whereas, in older subjects and particularly in those in whom calcified plaque was present, there was marked spatial and temporal variation in circumferential strain (Figure 1).
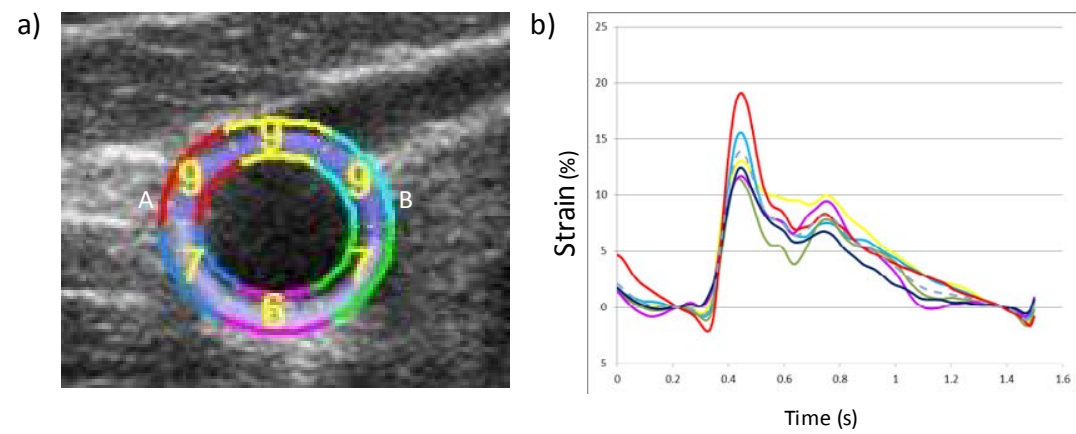

Figure 1: Variation in strain around the circumference of the common carotid artery

a) Circumferential strain is measured in six $60^{\circ}$ colour coded segments.

b) Strain curves in a young health subject with no evidence of calcification.

c) Strain curves in an older subject with calcified plaque elsewhere in the carotid. Older subjects show marked variation in peak circumferential strain; older subjects with calcification show marked variation in time to peak circumferential strain.

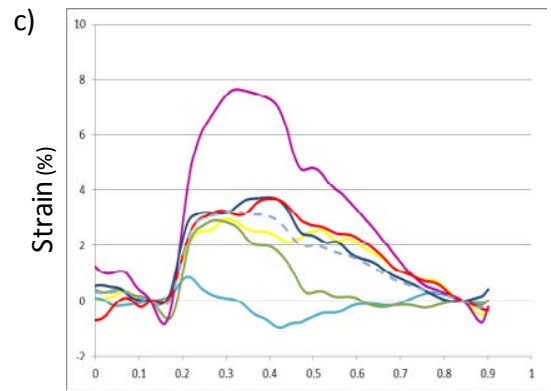

Time (s) 
Citation: Wang J, Spector T, Chowienczyk PJ, Jiang B (2015) A Novel Speckle Tracking Technique for Investigating Regional Motion of the Carotid Wall: Spatio-temporal Variation in Distension Associates with Presence of Calcified Plaque. J Vasc Med Surg 3: 233. doi:10.4172/23296925.1000233

\begin{tabular}{|c|c|c|c|c|}
\hline Measure & Total & Without plaque & With plaque & $\mathbf{P}$ \\
\hline Age & $61.50(10.18)$ & $59.73(10.58)$ & $65.85(7.65)$ & 0.02 \\
\hline Male, $\mathrm{n}(\%)$ & $32(13)$ & $23(13)$ & $9(12)$ & 0.92 \\
\hline BMI & $27.35(5.68)$ & $27.57(6.19)$ & $26.81(4.46)$ & 0.33 \\
\hline Current Smoker, $\mathrm{n}(\%)$ & $22(9)$ & $16(9)$ & $7(8)$ & 0.86 \\
\hline Hypertension, n (\%) & $79(32)$ & $50(28)$ & $29(39)$ & 0.08 \\
\hline Type 2 Diabetes, $\mathrm{n}(\%)$ & $11(4)$ & $7(4)$ & $4(5)$ & 0.58 \\
\hline SBP $(\mathrm{mmHg})$ & $126.98(15.44)$ & $124.42(16.17)$ & $133.56(14.22)$ & 0.47 \\
\hline DBP $(\mathrm{mmHg})$ & $73.79(8.44)$ & $73.70(8.52)$ & $74.02(8.27)$ & 0.69 \\
\hline Pulse Pressure(mmHg) & $53.20(12.90)$ & $50.72(11.54)$ & $59.54(14.10)$ & 0.11 \\
\hline HR (bpm) & $63.62(9.76)$ & $63.62(9.81)$ & $63.62(9.70)$ & 0.78 \\
\hline PWV( m/s) & $9.42(1.98)$ & $9.10(1.85)$ & $10.25(2.09)$ & 0.23 \\
\hline IMT (mm) & $0.71(0.13)$ & $0.70(0.14)$ & $0.74(0.17)$ & 0.03 \\
\hline
\end{tabular}

Table 1: Subject characteristics according to presence or absence of sub-clinical plaque. Values are mean (SD) or numbers (\%). BMI, body mass index; SBP systolic blood pressure; DBP, diastolic blood pressure; HR, heart rate; PWV, pulse wave velocity; IMT, intima-media thickness.

In subjects scanned on 2 occasions mean values of $\mathrm{VCS}_{\mathrm{p}}$ and $\mathrm{VCS}_{\mathrm{tp}}$ were $2.9 \pm 0.34 \%$ and $68.8 \pm 4.9 \mathrm{~ms}$ (means $\pm \mathrm{SE}$ ) and the mean differences of the measurements on the two occasions were $0.10 \pm 0.37 \%$ and $-4.0 \pm 0.10 .0 \mathrm{~ms}$ (means $\pm \mathrm{SD}$ ) for $\mathrm{VCS}_{\mathrm{p}}$ and $\mathrm{VCS}_{\mathrm{tp}}$ respectively giving coefficients of variation of $12.8 \%$ and $14.6 \%$ for VCS $\mathrm{V}_{\mathrm{p}}$ and $\mathrm{VCS}_{\mathrm{tp}}$ respectively.

VCS was correlated with age, presence of hypertension and weakly with serum lipids but not with presence of plaque (on univariate or mutivariate analysis, Table 2). Similar results were observed when considering variation of the ratio of strain to $\mathrm{cPP}$ (i.e., regional distensibility) or using values of strain normalized for mean strain (data not shown). By contrast, $\mathrm{VCS}_{\mathrm{tp}}$ was significantly correlated with age and calcified plaque (on univariate or mutivariate analysis, Table 2 ) as well as with body mass index and negatively correlated with heart rate (Table 2). The relationship between $\mathrm{VCS}_{\mathrm{tp}}$, age and presence of calcified plaque remained significant when all confounding factors were forced into the regression model (Table 2) and, furthermore, remained significant when any or all of IMT, average carotid distensibility and cfPWV were incorporated in the regression model (each $\mathrm{P}<0.001$ ).

\section{Discussion}

Elasticity of the carotid artery is usually assessed by measuring radial distension of the artery in a single transverse-plane dimension, with the assumption that elastic properties at a given plane through the cross-section of the artery are homogeneous and that strain around the circumference of the artery is constant [11,12]. 2-D speckle tracking imaging originally developed to study regional myocardial wall motion can be used to assess the arterial strain. Initial studies have focused on global strain (i.e., total circumferential strain) or peak segmental strain [13-15]. However dyssncrony of carotid wall strain has been demonstrated in patients with Marfan's syndrome [16].

The novel finding of the present study is that whilst 2-dimensional cross-plane circumferential strain is similar in young healthy subjects, marked heterogeneity exists in older subjects such that there is marked spatio-temporal variation of circumferential strain according to angular position around the circumference of the artery. Such variation in strain will increase shearing forces within the arterial wall and is thus likely to increase the propensity to plaque rupture [8-17]. We found that this variation in strain increased with age and with the presence of calcified plaque elsewhere within the artery. It is notable that calcified plaque is associated both with increased integrative measures of arterial stiffness, as measured by cfPWV $[18,19]$ and, except in subjects with advanced plaques $[20,21]$ (where calcification may confer stabilisation) [3], calcification is associated with increased risk of cardiovascular events $[1,3,4]$. Thus calcified plaque may be a marker for a heterogenous process of arterial stiffening, not necessarily limited to the site of macroscopically evident calcified atherosclerotic plaque that leads to spatio-temporal variation in arterial distension and distensibility and wall stress predisposing to plaque rupture.

There are two main implications of our findings. First, measures of distensibility obtained by a single transverse plane of the artery may be unrepresentative of the average distensibility of the artery, potentially impacting on the reliability of the measure in risk stratification. This could explain why, although correlated with cfPWV, measures of carotid distensibility are less predictive of cardiovascular events than those of cfPWV. Secondly, the indices of regional variability that we have described might provide sensitive measures of risk of cardiovascular events, acting as marker of arterial disease and/or predisposition to plaque rupture. Prospective outcome studies will, however, be required to test this hypothesis. They may also provide measures of structural change that are less dependent on blood pressure than existing measures of elasticity such as carotid distensibility and cfPWV. These existing measures are heavily dependent on transmural pressure and hence on mean arterial blood pressure distending the artery wall and transferring stress onto stiffer elements within the arterial wall. Variation in strain, however, may be less sensitive to absolute

\begin{tabular}{|l|c|c|c|c|}
\hline & \multicolumn{2}{|c|}{ VCS $_{\mathbf{p}}$} & \multicolumn{2}{c|}{ VCS $_{\text {tp }}$} \\
\hline Model 1 & $\boldsymbol{\beta}$ & $\mathbf{P}$ & $\boldsymbol{\beta}$ & $\mathbf{P}$ \\
\hline Mean peak CS & $\mathbf{0 . 1 8 5}$ & $<\mathbf{0 . 0 0 1}$ & & \\
\hline Mean time to peak CS & - & - & -0.032 & 0.245 \\
\hline Age & $\mathbf{0 . 0 3 0}$ & $\mathbf{0 . 0 0 2}$ & $\mathbf{0 . 7 2 1}$ & $<0.001$ \\
\hline Sex & -0.231 & 0.425 & -2.829 & 0.500 \\
\hline BMI & 0.15 & 0.379 & $\mathbf{0 . 8 0 1}$ & $\mathbf{0 . 0 0 2}$ \\
\hline Smoker & 0.246 & 0.433 & 4.557 & 0.337 \\
\hline Hypertension & $\mathbf{0 . 5 6 2}$ & $\mathbf{0 . 0 0 8}$ & -2.788 & 0.382 \\
\hline Diabetes & 0.068 & 0.877 & 9.676 & 0.151 \\
\hline MAP & 0.004 & 0.651 & 0.226 & 0.167 \\
\hline CPP & -0.002 & 0.848 & 0.070 & 0.660 \\
\hline Heart rate & -0.008 & 0.437 & $-\mathbf{0 . 5 2 7}$ & $\mathbf{0 . 0 0 2}$ \\
\hline Total cholesterol & $\mathbf{0 . 1 9 4}$ & $\mathbf{0 . 0 4 6}$ & 1.806 & 0.220 \\
\hline HDL & $\mathbf{- 0 . 5 7 0}$ & $\mathbf{0 . 0 2 1}$ & 0.665 & 0.856 \\
\hline Triglycerides & $\mathbf{0 . 5 1 2}$ & $\mathbf{0 . 0 3 1}$ & -0.518 & 0.885 \\
\hline Plaque & 0.142 & 0.549 & 1.950 & 0.588 \\
\hline Calcified plaque & 0.372 & 0.241 & $\mathbf{1 2 . 6 8 1}$ & $\mathbf{0 . 0 0 9}$ \\
\hline & & & & \\
\hline Model 2 & & & & \\
\hline Mean peak CS & $\mathbf{0 . 1 8 5}$ & $<\mathbf{0 . 0 0 1}$ & - & - \\
\hline Age & $\mathbf{0 . 0 3 4}$ & $<\mathbf{0 . 0 0 1}$ & $\mathbf{0 . 7 3 5}$ & $<\mathbf{0 . 0 0 1}$ \\
\hline BMI & - & - & $\mathbf{0 . 6 7 0}$ & $\mathbf{0 . 0 0 3}$ \\
\hline Hypertension & $\mathbf{0 . 6 0 8}$ & $\mathbf{0 . 0 0 2}$ & - & - \\
\hline MAP & - & - & $\mathbf{0 . 2 5 6}$ & $\mathbf{0 . 0 1 4}$ \\
\hline Heart rate & - & - & $-\mathbf{0 . 4 4 4}$ & $\mathbf{0 . 0 0 1}$ \\
\hline Total cholesterol & $\mathbf{0 . 1 9 6}$ & $\mathbf{0 . 0 3 1}$ & - & - \\
\hline HDL & $\mathbf{- 0 . 5 7 0}$ & $\mathbf{0 . 0 1 2}$ & - & - \\
\hline Triglycerides & $\mathbf{- 0 . 4 5 2}$ & $\mathbf{0 . 0 3 4}$ & - & - \\
\hline Calcified plaque & - & - & $\mathbf{1 4 . 1 8 1}$ & $<\mathbf{0 . 0 0 1}$ \\
\hline Tale & - & & & \\
\hline
\end{tabular}

Table 2: Multivariate analysis of relation between variation in circumferential strain measured by standard deviation of segmental peak strain (VCSp) and time of peak strain (VCStp) to risk factors and characteristics of plaque.

Model 1: all confounding variables entered.

Model 2: stepwise backward regression (CS, circumferential strain; BMI, body mass index; MAP, mean arterial pressure; CPP, central pulse pressure). 
Citation: Wang J, Spector T, Chowienczyk PJ, Jiang B (2015) A Novel Speckle Tracking Technique for Investigating Regional Motion of the Carotid Wall: Spatio-temporal Variation in Distension Associates with Presence of Calcified Plaque. J Vasc Med Surg 3: 233. doi:10.4172/23296925.1000233

distension and distensibility as suggested by results from the present study in which $\mathrm{VCS}_{\text {tp }}$ was unrelated to blood pressure components. This is a potentially important property since it might permit the distinction between interventions that specifically ameliorate arterial stiffening through a direct action on the arterial wall and those that do so indirectly simply by lowering blood pressure.

In conclusion, older subject, particularly those with evidence of calcified arterial plaque exhibit marked regional variation in carotid wall distension likely to predispose to plaque rupture. Measures of regional wall variation are repeatable and prospective studies to determine their potential in risk stratification and as a target for cardiovascular interventions are merited.

\section{Funding}

This work was supported by British Heart Foundation Special Project grant SP/12/4/29573. The authors acknowledge support from the National Institute for Health Research (NIHR) Clinical Research Facility at Guy's and St Thomas' NHS Foundation Trust and NIHR Biomedical Research Centre based at Guy's and St Thomas' NHS Foundation Trust and King's College London.

\section{Disclosures}

No conflict of interests to disclose.

\section{Novelty and Significance}

\section{What's new}

Wall speckle-tracking the carotid artery reveals marked spatiotemporal variation in distension of the carotid artery that associates with calcification.

\section{What's relevant}

Spatio-temporal variation in distension of the carotid artery could predispose to plaque rupture and may act as a marker of arterial wall stiffening that is independent of blood pressure.

\section{Summary}

Spatio-temporal variation in distension of the carotid artery associates with ageing and arterial calcification. It may predispose to plaque rupture and act as a marker of arterial wall stiffening that is.

\section{References}

1. Wattanakit K, Folsom AR, Chambless LE, Nieto FJ (2005) Risk factors for cardiovascular event recurrence in the Atherosclerosis Risk in Communities (ARIC) study. Am Heart J 149: 606-612.

2. Naqvi TZ, Lee MS (2014) Carotid intima-media thickness and plaque in cardiovascular risk assessment. JACC Cardiovasc Imaging 7: 1025-1038.

3. Prabhakaran S, Singh R, Zhou X, Ramas R, Sacco RL, et al. (2007) Presence of calcified carotid plaque predicts vascular events: The Northern Manhattan Study. Atherosclerosis 195: e197-201.

4. Komorovsky R, Desideri A, Coscarelli S, Cortigiani L, Tonello D, et al. (2005)
Prognostic implications of sonographic characteristics of carotid plaques in patients with acute coronary syndromes. Heart 91: 819-820.

5. Vlachopoulos C, Aznaouridis K, Stefanadis C (2010) Prediction of cardiovascular events and all-cause mortality with arterial stiffness: A systematic review and meta-analysis. J Am Coll Cardiol 55: 1318-1327.

6. Mattace-Raso FU, van der Cammen TJ, Hofman A, van Popele NM, Bos ML, et al. (2006) Arterial stiffness and risk of coronary heart disease and stroke: The Rotterdam Study. Circulation 113: 657-663.

7. Leone $\mathrm{N}$, Ducimetière $\mathrm{P}$, Gariépy J, Courbon D, Tzourio C, et al. (2008) Distension of the Carotid Artery and Risk of Coronary Events: The Three-City Study. Arteriosclerosis, Thrombosis, and Vascular Biology 28: 1392-1397.

8. Cheng GC, Loree HM, Kamm RD, Fishbein MC, Lee RT (1993) Distribution of circumferential stress in ruptured and stable atherosclerotic lesions. A structural analysis with histopathological correlation. Circulation 87: 1179-1187.

9. Richardson PD, Davies MJ, Born GV (1989) Influence of plaque configuration and stress distribution on fissuring of coronary atherosclerotic plaques. Lancet 2: $941-944$.

10. Lee RT, Grodzinsky AJ, Frank EH, Kamm RD, Schoen FJ (1991) Structuredependent dynamic mechanical behavior of fibrous caps from human atherosclerotic plaques. Circulation 83: 1764-1770.

11. Brands PJ, Hoeks AP, Willigers J, Willekes C, Reneman RS (1999) An integrated system for the non-invasive assessment of vessel wall and hemodynamic properties of large arteries by means of ultrasound. Eur $\mathrm{J}$ Ultrasound 9: 257-266.

12. Laurent S, Cockcroft J, Van Bortel L, Boutouyrie P, Giannattasio C, et al. (2006) Expert consensus document on arterial stiffness: Methodological issues and clinical applications. European Heart Journal 27: 2588-2605.

13. Bjällmark A, Lind B, Peolsson M, Shahgaldi K, Brodin L, et al. (2010) Ultrasonographic strain imaging is superior to conventional non-invasive measures of vascular stiffness in the detection of age-dependent differences in the mechanical properties of the common carotid artery. European Heart Journal - Cardiovascular Imaging 11: 630-636.

14. Widman E, Caidahl K, Heyde B, D'hooge J, Larsson M (2015) Ultrasound Speckle Tracking Strain Estimation of in-Vivo Carotid Artery Plaque with inVitro Sonomicrometry Validation. Ultrasound in Medicine \& Biology 41: 77-88.

15. Yang EY, Dokainish H, Virani SS, Misra A, Pritchett AM, et al. (2011) Segmental analysis of carotid arterial strain using speckle-tracking. J Am Soc Echocardiogr 24: 1276-1284.

16. Yang WI, Shim CY, Cho IJ, Chang HJ, Choi D, et al. (2010) Dyssynchronous systolic expansion of carotid artery in patients with marfan syndrome. J Am Soc Echocardiogr 23: 1310-1316.

17. Wang KL, Cheng HM, Chuang SY, Spurgeon HA, Ting CT, et al. (2009) Central or peripheral systolic or pulse pressure: which best relates to target organs and future mortality? J Hypertens 27: 461-467.

18. Zureik M, Bureau JM, Temmar M, Adamopoulos C, Courbon D, et al. (2003) Echogenic carotid plaques are associated with aortic arterial stiffness in subjects with subclinical carotid atherosclerosis. Hypertension 41: 519-527.

19. Cecelja M, Jiang B, Bevan L, Frost ML, Spector TD, et al. (2011) Arteria stiffening relates to arterial calcification but not to noncalcified atheroma in women. A twin study. J Am Coll Cardiol 57: 1480-1486.

20. Seeger JM, Barratt E, Lawson GA, Klingman N (1995) The relationship between carotid plaque composition, plaque morphology, and neurologic symptoms. J Surg Res 58: 330-336.

21. Shaalan WE, Cheng H, Gewertz B, McKinsey JF, Schwartz LB, et al. (2004) Degree of carotid plaque calcification in relation to symptomatic outcome and plaque inflammation. J Vasc Surg 40: 262-269. 